Open Peer Review on Qeios

\title{
The effects of offering a disposable EC versus NRT on smoking cessation outcomes: a retrospective community pharmacy study
}

\author{
Kirstie Soar ${ }^{1}$, Jay Doshi², James Cameron \\ 1 University of East London \\ 2 National Health Service
}

Funding: The author(s) received no specific funding for this work.

Potential competing interests: KS has provided research consultancy to PharmStrat Ltd a healthcare consulting company. JD has no COI. JC operates stop smoking clinics for The Quit Clinic in Birmingham and Grovehill and Woodhall Pharmacies and has also provided healthcare consulting for UK pharmacies and advice regarding medical license application to Evapo and Thornton and Ross.

\section{Abstract}

Background: E-cigarettes (EC) are now a viable alternative to traditional pharmacotherapies for supporting a quit attempt, with increasing evidence demonstrating their efficacy for smoking cessation and are the most popular quitting aid within the UK. However, their effectiveness within a community pharmacy stop smoking service (SSS) has received limited attention.

Objectives: To measure the effectiveness of offering a disposable EC with and/or without nicotine replacement therapy (NRT) compared to NRT alone, on both 4-6 week and 12-week quit rates in adult smokers attending a community pharmacy.

Methods: A non-randomised between subjects design was employed involving 1488 smokers willing to quit $(835=$ female; age $=41.98, \mathrm{SD}=11.31)$. Smokers choose either an $E C, E C+N R T$ (EC condition) or NRT alone (NRT condition), alongside standard behavioural support.

Results: Overall quit rates at 4- weeks were $57 \% ; 56 \%$ for those in the EC condition and $61 \%$ for those in the NRT condition. At 12 -weeks overall quit rates 30\%; 30\% in the EC condition and 32\% in the NRT. There were no significant differences between conditions at either follow up period. Conclusions: Four-week quit rates were in line with the national average. Offering a disposable EC showed to have comparable quit rates with those who received only NRT both at 4-6 and 12 weeks and that given the choice a significantly large number of smokers choose to use an EC within their cessation attempt within a community pharmacy setting

\section{INTRODUCTION}

Smoking prevalence has steadily declined in the UK over recent decades years (Opazo Breton et al 2021), with recent estimates of only $14.2 \%$ of adults classified as current regular cigarette smokers (Smoking 
Toolkit, 2021). This is largely due to reduced smoking uptake in under 25 's, and to a lesser extent smoking cessation after this age (Opazo Breton et al 2021). However, there remains 5.9 million smokers, and many are in particular disadvantaged groups. Given cigarette smoking is still the single leading cause of preventable illness and death, with 74,600 deaths caused by smoking each year (NHS Digital, 2020), reducing smoking uptake and in particular increasing smoking cessation rates therefore remains an urgent priority $(\mathrm{DOH}, 2017)$.

The Governments ambition for England is to be Smokefree by 2030, 10 years ahead of the EU (APPG, 2021). With this comes the subsequent economic and health benefits, which will be felt mostly amongst the disadvantaged groups and in the most disadvantaged areas (APPG, 2021). However, to reach this target the UK need to reduce smoking by two thirds in the next 10 years, specifically $3 / 4$ of those smokers in manual and routine occupations. These targets can only be achieved by motivating smokers to make a quit attempt using the most effective quitting aids and maximising the proportion of successful quits per quit attempt.

E-cigarettes are a viable alternative to traditional pharmacotherapies for supporting a quit attempt and the ccurrent guidance on stop smoking interventions and services from NICE and the Tobacco Control plan (2017) recommends research evaluating the effectiveness and cost-effectiveness of EC particularly among disadvantaged groups. Evidence for the efficacy of EC for smoking cessation is accumulating. The most recent Cochrane review which includes 29 randomised control trials (RCTs) involving 12,804 smokers, indicates that EC increases quit rates with no additional adverse effects compared to NRT and behavioural support/no treatment (Hartman-Boyce, 2021). They also conclude that with moderate certainty, use of nicotine containing EC were twice as effective as NRT for long term smoking cessation. And whilst the debate about their long-term safety remains, it has been agreed that the risks of EC are considerably less than continued smoking (McNeill et al, 2015).

EC are also the most popular choice in smokers wishing to quit (Smoking Toolkit, 2021) and rates of use by different socio-economic groups are starting to converge (Kock et al, 2018). They are less expensive in the UK than smoking in the long term, however they do come with initial startup costs which may deter smokers from using them, especially those on lower incomes (Thirlway, 2019), yet for SSS they have been shown to be more cost-effective compared with NRT (Li et al, 2019).

Many Stop Smoking Services (SSS) have been reluctant to include EC among their treatment options because data on their effectiveness compared with the licensed medications has been lacking, until recently. Although all SSS offer NRT and behavioural support, only $11 \%$ of local authority funded SSS in England offer EC (ASH, 2019), whereas others who consider themselves 'e-cigarette friendly' offer support and advice around EC use in line with NICE guidelines (NICE, 2018) which currently advises 'people who smoke should not be discouraged from switching to e-cigarettes and as a result continue to smoke'. 
Stop Smoking services are often delivered by community pharmacies, offering the traditional over the counter nicotine replacement therapy (NRT), advice and in some cases behavioural support, however with varied success (Bauld et al, 2010; Sinclair, Bond \& Stead, 2004). Given there is a a renewed push for the Government's next Tobacco Control Plan (due 2021) to meet their commitment of "make the route to medicinal licensing, for EC fit for purpose to allow e-cigarettes to be authorised for NHS prescription" [recommendation 11] (APPG, 2021), with the aim to have a range of safe and effective EC products on offer by 2022. In addition to the commitment to make those medically licensed EC a first line NRT and provide funding to make them available on prescriptions for those smokers wanting to quit (APPG, 2021), suggests community pharmacies could play an even more important role in delivering successful smoking services in the future. Indeed, a recent exploratory study assessing the effectiveness of offering an EC, within a community pharmacy setting in Hertfordshire, England demonstrated positive results. Onehundred and fifteen smokers were offered the choice of an EC, EC and NRT or NRT alone, alongside standard behavioral support. Those smokers who opted for an EC alone and/or with NRT were more likely to report smoking abstinence at 4-6 weeks (Cox et al, 2019). However, longer term abstinence needs to be ascertained, given an observational study has shown 4-week pharmacy CO validated quit rates with NRT to drop over 12 -momths from $22 \%$ to $4 \%$.

The current study aims to build on the findings of Cox et al (2019) and presents a study which measures the effectiveness of offering a disposable EC with, or without NRT, and standard behavioural support within a community pharmacy in Birmingham, England over a longer period. The study employed a larger sample of smokers, from an area that is likely to attract smokers from potentially different socio-economic backgrounds. The primary outcome was to assess self-report smoking cessation rates at both 4-6 weeks and 12-weeks, in those using an EC over a 12- week program, compared to NRT alone.

\section{METHODS}

\section{Participants}

One thousand, four hundred and eighty-eight smokers (835 $(56 \%)=$ female; age $=41.98, \mathrm{SD}=11.31$ ) who showed a willingness to quit were recruited mainly through the SSS social media channels, using a variety of platforms. The recruitment content varied from advertising of the clinic and services, to use of digital ads from Stoptober and No Smoking Day campaigns and NHS health articles relating to Pregnancy, COVID, COPD and Asthma. To note, the offer of an e-cigarette was not advertised due to regulatory restrictions. Participants were included in the trial if they were a smoker, over 18 years of age, living, working or their GP based within Birmingham electoral wards (as determined by post codes). Smoking status was selfreported only. Due to Covid-19 social distancing measures smoking status could not be verified by CO 
monitoring. Exclusion criteria included pregnancy and/or breastfeeding, $<18$ or $>80$ years old and were requesting or currently using varenicline or buprenorphine were excluded from the service and advised to contact their GP. All participants gave informed consent. The study was conducted in accordance with the Declaration of Helsinki and Public Health England protocol and recorded in the NHS reporting system, PharmaOutcomes.

\section{Design}

A non-randomized retrospective cohort, between subjects design study was employed. Participants chose treatment condition; either EC with or without NRT (EC condition) or combination NRT alone (NRT condition). The primary outcomes were self-reported smoking abstinence at 4-6 weeks follow up, and at 12- week follow up. Objective verification of smoking abstinence was not possible due to Covid-19 social distancing measures. Participants were considered as non-quitters if they dropped out, were lost to follow up or reported smoking.

\section{Measures}

Socio-demographic measures were obtained from all participants (age, gender, ethnicity, occupational status, levy status and current medical history). Current smoking status was ascertained by self-report only, due to Covid-19 restrictions. Cigarette dependence was assessed by the Fagerstrom test of Cigarette dependence (FTCD; Fagerstrom, 2011). The FTCD is a standard well used measure of the severity of nicotine dependence, based on 6 items which evaluate the quantity of cigarette consumption, use and dependence. Items are scored on either 0 to 1 on yes/no items or 0 to 3 or multiple-choice items. A total dependence score ranges from 0-10; the higher the score the higher the level of dependence.

Participants previous use of NRT use was also recorded by indicating which types and combinations of NRT they had previously used in a quit attempt, as well as how they became aware of the stop smoking service (service awareness) from a choice of 8 options (GP, friend or relative, other health care profession, advertising, word of mouth, NHS health checker or other).

At each follow up (4-6 week and 12-week) self-reported abstinence rates were recorded and alongside confirmation of support they were continuing to use (if any) in their cessation attempt.

The NRT options available to participants either alongside an EC or on their own were 16-hour patches (10, 15 or $25 \mathrm{mg}$ ), 24 hour patches $(7,14,21 \mathrm{mg}$ ) gum (icy white mint/fruit flavoured. $2 \mathrm{mg}$ or $4 \mathrm{mg}$ ), mouth spray $1 \mathrm{mg} / \mathrm{spray}$ (fresh mint or cool berry), inhalator $(15 \mathrm{mg})$, nasal spray $(10 \mathrm{ml})$, micotabs $(2 \mathrm{mg})$ or lozenges (mint flavoured, $2 \mathrm{mg}$ or $4 \mathrm{mg}$ ). 
A disposable EC (Mylo Go) was used. Each EC contained $1.2 \mathrm{ml}$ of e-liquid, which consisted of $18 \mathrm{mg} / \mathrm{ml}$ of nicotine, with a choice of 4 flavours: tobacco, menthol, blueberry, and raspberry. Participants were permitted to switch between flavours in accordance with documented vaping practices. The Mylo Go EC was offered for the 12-week program. A disposable EC was chosen as it does not require purchase of coils and liquids, which would be difficult for many patients to acquire and apply, especially during the Covid-19 pandemic.

\section{Procedure}

Following recruitment and screening, appointments were made and conducted by telephone and/or text (due to Covid-19 restrictions). Following verbal consent socio-demographics, smoking measures and service awareness were recorded. Participants then received behavioural advice in terms of their addiction to nicotine, the benefits of NRT and/or e cigarettes or therapy and the different types of NRT support available. They were then offered several therapy options and requested to select which NRT they required and if EC, which of the four flavours. Patients were then given 2 weeks supply of NRT and/or EC and received these by post or through their local pharmacy. For participants choosing to use an EC, 4 were supplied at the initial assessment for a two-week period (i.e., 2 per week), and 2 thereafter (i.e., 1/week). A telephone appointment was agreed with their stop smoking advisor every 2 weeks up to 12 weeks. Patients therefore were offered a maximum of seven appointments in total. Outcome measures were recorded at the 4-6 week and 12-week appointments.

EC and NRT was offered free of charge if the smoker qualified for free prescriptions; other patients paid a one-off charge of one prescription fee All smokers were offered the full 12-week standard smoking cessation support with behavioural support. There were no other financial incentives offered.

\section{Data analysis}

One percent $(n=19)$ of participants elected to just use an EC in their quit attempt, as such their data has been included in the EC and NRT group. There were a small number of participants ( $4 \% ; n=55)$ who chose to use NRT only at baseline, however over the course of the 12-week program, for one reason or another they chose to try an e-cigarette. Therefore, participants have been grouped and data analysed based on what form of support they used over the duration of the 12-week program i.e., whether they used NRT only throughout their quit attempt (NRT only) or tried an EC with or without NRT (EC). All data was analysed in SPSS 26. Differences between conditions (EC versus NRT) on demographics, smoking related data and quit status were calculated using $\mathrm{Chi}^{2}$ tests for categorical variables and independent $\mathrm{t}$-tests for continuous data. Only descriptive data on previous pharmacy support is presented due the expected frequency $<5$ on more than $20 \%$ of the cases therefore a $\mathrm{Chi}^{2}$ test would not have been statistically viable. All differences were considered significant if $p \geq 0.05$. 


\section{RESULTS}

\section{Baseline Sociodemographic data}

One thousand, four hundred and eight-eight participants were recruited to the trial. At baseline twohundred and thirteen (14\%) opted to receive NRT only, 1,256 (84\%) opted for the EC alongside one other form of NRT and 19 (1\%) opted for EC only. Table 1 presents sociodemographic data for trial participants as a sample and by condition (EC versus NRT). There were significantly more females than males, opting for an EC compared to only NRT in their cessation attempt $\left[\mathrm{Chi}^{2}(1)=3.91, p=0.05\right]$. Participants did not differ significantly by age, ethnicity, occupational status, levy status or whether they had a medical condition across treatment conditions.

\section{Smoking Related Data}

Table 2 presents smoking related data for the whole sample and by condition. Participants mean Fagerstrom scores $(m=6.17, S D=1.98)$ indicated very high addiction to tobacco products, however there were no significant differences in dependence scores between EC and NRT conditions, nor were there significant differences between type of tobacco abuse or reported previous cessation support. There was a significant difference in the number of people who reported how they found out about the service depending on whether they were chose an EC or NRT only in their cessation attempt $\left[\mathrm{Chi}^{2}(7)=28.13, p<\right.$ $0.001]$.

At 4 weeks, 843 (57\%) participants self-reported no longer smoking. Quit rates were slightly higher for those using NRT (61\%), compared to EC (56\%), however this difference was not statistically significant $\left[\mathrm{Chi}^{2}(1)=1.21, p=0.27\right]$. At 12-week follow-up, 445 (30\%) self-reported a quit status, with no statistical difference in quit rates between those receiving NRT (32\%) versus EC $(30 \%)$, $\left[\mathrm{Chi}^{2}(1)=0.48, \mathrm{p}=0.49\right]$.

There were no observable differences between conditions on previously reported pharmacy support (table 2 ). There was a significant difference by condition in reporting of service awareness $\left[\mathrm{Chi}^{2}(7)=28.13\right.$, $p<0.001$ ], with a higher percentage of those in the EC condition being aware of the service via advertising compared to those in the NRT, and a higher percentage of those in the NRT being aware of the service because they were re-enrolling with the SSS.

Table 1: Participant data at baseline, total sample and by condition (EC v NRT)

\begin{tabular}{|c|c|c|c|c|c|}
\hline & $\begin{array}{l}\text { Total } \\
(n=1488)\end{array}$ & $\begin{array}{l}E C \\
(n=1330)\end{array}$ & \multicolumn{2}{|c|}{$\begin{array}{l}\text { NRT } \\
(n=158)\end{array}$} & $\mathbf{P}$ \\
\hline & Mean (SD) & Mean & Mean & Range & \\
\hline \multirow[t]{2}{*}{ Age (years) } & 41.98 (11.25) & 41.81 (11.71) & 43.46 & $(22-72)$ & 0.08 \\
\hline & $\%$ & $\%$ & $\mathbf{N}$ & $\%$ & \\
\hline
\end{tabular}




\section{Gender}

Male

Female

Ethnicity

Any other ethnic group

Arab

Asian

Black

Mixed

Not stated

Prefer not to say

White

\section{Occupational Status}

Unemployed

Home Carer

Managerial and Professional

Intermediate

Routine and Manual

Retired

Sick or disabled

Full time student

Unknown

\begin{tabular}{l|l|l|l|l|l|}
653 & 43.9 & 572 & 43 & 81 & 51 \\
\hline 835 & 56.1 & 758 & 57 & 77 & 49
\end{tabular}

0.38

\begin{tabular}{|c|c|c|c|c|c|}
\hline 44 & 3 & 38 & 2.9 & 6 & 3.8 \\
\hline 1 & 0.1 & 1 & 0.1 & 0 & 0 \\
\hline 195 & 13 & 183 & 13.8 & 12 & 7.6 \\
\hline 5 & 0.3 & 5 & 0.4 & 0 & 0 \\
\hline 54 & 3.6 & 50 & 3.8 & 4 & 2.5 \\
\hline 132 & 8.9 & 117 & 8.8 & 15 & 9.5 \\
\hline 6 & 0.4 & 6 & 0.5 & 0 & 0 \\
\hline 1051 & 70.6 & 930 & 69.9 & 121 & 76.6 \\
\hline
\end{tabular}

$<0.001$

$\begin{array}{lllllll}553 & 37.2 & 515 & 38.7 & 38 & 24.1\end{array}$

$\begin{array}{lllllll}72 & 4.8 & 64 & 4.8 & 8 & 5.1\end{array}$

\begin{tabular}{l|l|l|l|l|l}
222 & 14.9 & 204 & 15.3 & 18 & 11.4
\end{tabular}

$\begin{array}{lllllll}161 & 10.8 & 148 & 11.1 & 13 & 8.2\end{array}$

$\begin{array}{llllll}204 & 13.7 & 181 & 13.6 & 23 & 14.6\end{array}$

$\begin{array}{llllll}54 & 3.6 & 46 & 3.5 & 8 & 5.1\end{array}$

$\begin{array}{lllllll}33 & 2.2 & 29 & 2.2 & 4 & 2.5\end{array}$

\begin{tabular}{l|lllll}
10 & .7 & 10 & 0.8 & 0 & 0
\end{tabular}

\begin{tabular}{l|l|l|l|l|l}
179 & 12.0 & 133 & 10.0 & 46 & 29.1
\end{tabular}

0.05

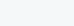

\section{Levy Status}

0.72

16,17 or 18 in full-time education

60 years of age or over

Valid maternity exemption certificate

Valid medical exemption certificate

Valid prescription pre-payment certificate

Gets Income Support or income related ESA

Gets income-based Jobseekers Allowance

Named on a current HC2 charges certificate

Entitled or named on valid NHS Tax Credit Exemption Certificate

N/A

Medical conditions (yes)

\begin{tabular}{|c|c|c|c|c|c|}
\hline 5 & .3 & 5 & 0.4 & 0 & 0 \\
\hline 78 & 5.2 & 68 & 5.1 & 10 & 6.3 \\
\hline 11 & .7 & 10 & 0.8 & 1 & 0.6 \\
\hline 226 & 15.2 & 199 & 15.0 & 27 & 17.1 \\
\hline 66 & 4.4 & 59 & 4.4 & 7 & 4.4 \\
\hline 419 & 28.2 & 384 & 28.9 & 32 & 22.2 \\
\hline 208 & 14.0 & 189 & 14.2 & 19 & 12.0 \\
\hline 6 & .4 & 5 & 0.4 & 1 & 0.6 \\
\hline 66 & 4.4 & 59 & 4.4 & 7 & 4.4 \\
\hline 403 & 27.1 & 352 & 26.5 & 51 & 32.3 \\
\hline 532 & 35.8 & 480 & 36.1 & 52 & 32.9 \\
\hline
\end{tabular}

Table 2: Smoking related data for the total sample and by condition (EC V NRT) 


\begin{tabular}{|c|c|c|c|c|c|c|c|}
\hline & \multicolumn{2}{|c|}{$\begin{array}{l}\text { Total } \\
(n=1488)\end{array}$} & \multicolumn{2}{|c|}{$\begin{array}{l}E C \\
(n=1330)\end{array}$} & \multicolumn{2}{|c|}{$\begin{array}{l}\text { NRT } \\
(n=158)\end{array}$} & \multirow[t]{2}{*}{$\mathbf{p}$} \\
\hline & Mean & SD & Mean & SD & Mean & SD & \\
\hline \multirow[t]{2}{*}{$\begin{array}{l}\text { FTCD Score } \\
\text { (at baseline) }\end{array}$} & 6.17 & 1.98 & 6.19 & 1.98 & 6.04 & 2.01 & 0.37 \\
\hline & $\mathbf{N}$ & $\%$ & $\mathbf{N}$ & $\%$ & $\mathbf{N}$ & $\%$ & \\
\hline Previous Pharmacy Support: & & & & & & & - \\
\hline None & 467 & 31.4 & 437 & 32.9 & 30 & 19 & \\
\hline EC only & 57 & 3.8 & 52 & 3.9 & 5 & 3.2 & \\
\hline EC \& NRT & 278 & 18.7 & 242 & 19.2 & 36 & 22.8 & \\
\hline EC \& Champix & 1 & .1 & 1 & .1 & 0 & 0 & \\
\hline EC, Champix \& NRT & 4 & 0.3 & 3 & 0.2 & 1 & 0.6 & \\
\hline EC, Varenicline \& NRT & 2 & .1 & 1 & .1 & 1 & 0.6 & \\
\hline NRT only & 603 & 40.5 & 523 & 39.3 & 80 & 50.6 & \\
\hline NRT \& Champix & 23 & 1.5 & 22 & 1.7 & 1 & 0.6 & \\
\hline NRT, Champix \& Zyban & 1 & .1 & 1 & .1 & 0 & 0 & \\
\hline NRT, Varenicline & 5 & 0.3 & 5 & 0.4 & 0 & 0 & \\
\hline Champix only & 45 & 3.0 & 42 & 3.2 & 3 & 1.9 & \\
\hline Varenicline only & 2 & .1 & 1 & .1 & 1 & 0.6 & \\
\hline Awareness of specific SSS & & & & & & & $<0.001$ \\
\hline Advertising & 1359 & 91.3 & 1229 & 92.4 & 130 & 82.3 & \\
\hline Re-enrolled onto SSS & 44 & 3.0 & 30 & 2.3 & 14 & 8.9 & \\
\hline Friend/Relative & 32 & 2.2 & 27 & 2.0 & 5 & 3.2 & \\
\hline Pharmacy & 24 & 1.6 & 20 & 1.5 & 4 & 2.5 & \\
\hline GP & 13 & .9 & 10 & 0.8 & 3 & 1.9 & \\
\hline Word of Mouth & 12 & .8 & 11 & 0.8 & 1 & 0.6 & \\
\hline Health Care Professional & 3 & .2 & 2 & 0.2 & 1 & 0.6 & \\
\hline Other & 1 & .1 & 1 & 0.1 & 0 & 0 & \\
\hline \multicolumn{8}{|l|}{ Number of quit attempts } \\
\hline 4 weeks & 843 & 57 & 747 & 56 & 96 & 61 & 0.27 \\
\hline 12 weeks & 445 & 30 & 394 & 30 & 51 & 32 & 0.49 \\
\hline
\end{tabular}

$E C=$ e-cigarette; $N R T=$ nicotine replacement therapy; SSS = Stop Smoking Service; GP = General Practitioner

\section{DISCUSSION}

The current study examined the effect of offering a disposable EC with, or without NRT and standard behavioural support in a community pharmacy, on smoking cessation outcomes at both 4-6 weeks and 12 
weeks, in a large sample of smokers. Offering a disposable EC showed to have comparable quit rates with those who received only NRT, with no significant differences between the two conditions at either follow up period. Overall quit rates at 4 - weeks were $57 \%$ of all smokers who attempted a cessation attempt; $56 \%$ for those in the EC condition and $61 \%$ for those in the NRT condition. Cessation rates that are comparable to national average (58.6\%; NHS digital $2020 / 21$ ). Overall, quit rates at 12 -weeks were $30 \%$ of all smokers who attempted a cessation attempt; $30 \%$ in the EC condition and $32 \%$ in the NRT. Providing evidence to suggest that longer term outcomes (12-weeks) in relation to offering an EC via a community pharmacy SSS are also comparable with NRT. It is also interesting to note that the drop in the quit rates between 4- and 12- weeks was less in the EC than the NRT which could be seen as a preliminary indication that the use of an EC alongside NRT may be more effective in the longer term, however this can only be determined with longer term follow up (e.g., 52 weeks) which is currently being monitored.

It is also worthy to note that cessation rates across all conditions are higher than seen in previous community pharmacy studies using NRT, at similar time points (Sinclair, Bond \& Stead, 2004; Bauld et al, 2011); high number of quits achieved with the equivalent of 2.5FTE stop smoking advisors, accounting for $176 \%$ of the average England Area Health Authority quit rates (see Soar, 2021). Therefore, the use of an offer of an EC as part of the SSS, provides encouraging support for the role in community pharmacies delivering SSS going forward.

The current study does indicate that the offer of an EC is considered attractive to those smokers who have accessed a SSS, given the vast majority (84\%) elected to use an EC either on its own or alongside another form of NRT within their cessation attempt. This preference for an EC in a smoking cessation attempt is consistent with the evidence (Smoking Toolkit, 2021), but also could be influenced by the advice and guidance offered by the smoking advisors. This guidance may also account for why only $1 \%$ chose to use an EC only, as participants were informed that two forms of NRT support are more effective than one in a cessation attempt prior to their choice (NCSCT, 2021). Regardless, being able to offer cessation aids which matches patients' preferences is expected to help with recruitment and compliance of patients within an SSS and ultimately increase the chances of a successful smoking cessation attempt.

Providing more opportunities and flexibility for smokers in a cessation attempt is considered beneficial (APPG, 2021) and this study supports this given that most opted for an EC, but also as evidenced over the course of the 12-week program, with 55 participants who originally elected to have NRT only, went on to try an EC at some point during their 12-week cessation attempt. This flexibility is something which is not documented or evidenced in RCTs and highlights the importance of community studies such as this.

Whilst the current study did not support the findings that offering an EC wasmore effective than NRT at 4- 
weeks, akin to that in the smaller community pharmacy study (Cox et al, 2019), this could be accounted for by, sample size and different socio-demographic profiles of participants. Participants in the current study were more likely to be unemployed with the majority on some form of financial reimbursement i.e., income support or job seekers allowance. That the offering an EC was as effective as NRT in this study, and given the good smoking cessation rates demonstrated, it highlights that such a cessation intervention is targeting and attracting those from disadvantaged groups - the current priority groups for smoking cessation (APPG, 2021).

An additional reason why the EC may not have been more effective than NRT in this current study relative to that shown in Cox et al (2019), is because of the nicotine content in the disposable EC was not comparable to other forms of NRT or even continued smoking. The stop smoking advisors observed that participants in the current study were reporting that the EC was not lasting the expected period allocated, thus could potentially increase the risk of relapse to smoking and effect the effectiveness of the EC as a cessation aid. The EC chosen for this trial was a Mylo Go disposable EC containing $1.2 \mathrm{ml}$ of e-liquid consisting of $18 \mathrm{mg} / \mathrm{ml}$ of nicotine. Those who elected to use an EC with or without another form of NRT were provided with 4 EC at the initial assessment for a two-week period (i.e., 2 per week), and 2 thereafter (i.e., 1/week) with the assumption that one disposable EC would last the patient between 1-3 days. Weekly nicotine content per week is considerably lower for the EC condition compared to other combination NRT scenarios (e.g., $12 \%$ that of a $25 \mathrm{mg}$ patch). EC nicotine content was also considerably lower compared to that if the participant continued to smoke (see Soar, 2021 for further details). Thus, participants electing to use an EC may not have been receiving comparable levels of nicotine to other forms of NRT offered. This could therefore have accounted for drop out and/or a return to smoking and potentially account for why the EC condition was not showing better effectiveness on quit rates relative to NRT, as seen in other previous studies (e.g., Cox et al, 2019). Thus, future community studies should consider providing an EC intervention with comparable nicotine content with other NRTs and evaluate subsequent impacts on cessation rates.

The current study is not without its limitations. Participants were not randomised to conditions, with smokers being able to choose which products to use following guidance from a stop smoking advisor, which is open to bias. Additionally, participants who choose to use an EC may have different motivations, believes and attitudes towards smoking and in particular ECs, which could also indirectly effect motivations to quit, but given comparable rates were seen in both conditions, this is unlikely to be a strong influence given the number of people in the EC condition. Secondly, smoking status (at baseline) and subsequent quit status (at follow ups) were not CO verified, however this was beyond the control of the study given Covid-19 social distancing measures. Thirdly, whilst data was available on those participants who moved from NRT only to using an EC $(n=55)$ during their cessation attempt, data was not available on participants who may have used NRT and EC interchangeably over their cessation attempt, and whether participants 
were still using either NRT or EC at both 4- and 12- weeks. Nor were routine data on choice of EC flavour recorded and what, if any changes were made over the course of the cessation attempt. These would be important to monitor given EC are not without their risks and evidence indicates such hazards my be dependent on flavours (e.g., Qu, Kim \& Szulejko, 2018; Holden et al, 2020).

Despite the limitations, the study has shown evidence in a community setting using a large sample, that offering an EC as an additional cessation aid can support smoking cessation rates comparable with that of NRT both at 4- and 12-weeks and that given the choice a significantly large number of participants choose to use an EC within their cessation attempt. Follow up at 52-weeks would be useful to determine whether effects are sustained or are better than NRT alone.

\section{REFERENCES}

All Party Parliamentary Group on Smoking and Health (APPG) (2021) Delivering a Smokefree 2030: The AllParty Parliamentary Group on Smoking and Health recommendations for the Tobacco Control Plan 2021. June 2021 https://ash.org.uk/wp-content/uploads/2021/06/APPGTCP2021.pdf

ASH (Action on Smoking and Health and Cancer Research UK) (2012). Many ways forward: Stop smoking services and tobacco control work in English local authorities. https://ash.org.uk/information-andresources/reports-submissions/reports/many-ways-forward/ Accessed on 05.10.21.

Cox S, Dawkins L, Doshi J, Cameron J (2019). Effects of e-cigarettes versus nicotine replacement therapy on short-term smoking abstinence when delivered at a community pharmacy. Addictive Behaviour Reports, 10, 100202.

Bauld L, Boyd KA, Briggs AH, Briggs AH, Chesterman J, Ferguson J, Judge K, Hiscock R (2010) One-year outcomes and a cost effectiveness analysis for smokers accessing group-based and pharmacy-led cessation services. Nicotine \& Tobacco Research, 13(2):135-145

DOH (2017) Smoke-free generation: tobacco control plan for England. https://www.gov.uk/government/publications/towards-a-smoke-free-generation-tobacco-controlplan-for-england Accessed 05 Oct 2021

Fagerstrom K (2011) Determinants of tobacco use and renaming the FTND to the Fagerstrom Test for cigarette dependence. Nicotine \& Tobacco Research. 14(1):75-8

Hartman-Boyce J, McRobbie H, Lindon N, Bullen C, Begh R, Theodoulou A, Notley C, Rigotti NA, Turner T, 
Butler AR, Fanshawe TR, Hajek P. (2021) Electronic cigarettes for smoking cessation. Cochrane Database of Systematic Reviews 2021, Issue 4. Art. No:CD010216. DOI:10.1002/14654858.CD010216.pub5

Holden LL, Truong L, Simonich MT, Tanguay RL (2020) Assessing the hazard of E-Cigarette flavor mixtures using zebrafish. Food and Chemical Toxicology. 136: 110945. https://doi.org/10.1016/i.fct.2019.110945.

Kimber C, Soar K \& Dawkins LE (2021). Changes in puffing topography and subjective effects over a 2week period in e-cigarette naïve smokers: effects of device type and nicotine concentrations. Addictive Behaviours.

Kock L, Shab L, West R, Brown J, (2018) E-cigarette use in England 2014-17 as a function of socio-economic profile. Addiction 114 (2), 294-303.

Li J, Hajek P, Pesola F, Wu Q, Phillips-Water A, Przulj D, Myers Smooth K, Bisal N, Sasieni P, Dawkins L, Ross L, Lukasz Goniewicz M, McRobbie H, Parrott S. (2019) Cost-effectiveness of e-cigarettes compared with nicotine replacement therapy in stop smoking services in England (TEC study): a randomized controlled trial. Additction, 115:507-517.

McNeil A, Brose L.S., Calder R, Hitchman S, Hajek P, McRobbie H. (2015) E-cigarettes: an evidence update. London: Public Health

England. https://assets.publishing.service.gov.uk/government/uploads/system/uploads/attachment_data/file/ 733022/Ecigarettes_an_evidence_update_A_report_commissioned_by_Public_Health_England_FINAL.pdf Accessed 05.10.21

NCSCT (2021) Combination nicotine replacement therapy (NRT). Briefing. August 2021. https://www.ncsct.co.uk/usr/pub/Combination\%20NRT\%202021.pdf Accessed 15.10.21.

NHS Digital, Statistics on Smoking: England2020. 2020: https://digital.nhs.uk/data-andinformation/publications/statistical/statistics-on-smoking/statistics-on-smoking-england2020. [Accessed 30 May 2021].

NICE guidelines (2018) https://www.nice.org.uk/guidance

Opazo Breton M, Gillespie D, Pryce R, Bogdanovica I, Angus C, Hernandez Alava M, Brennan A, Britton J (2021) Understanding long-term trends in smoking in England, 1972-2019: an age-period-cohort approach Addiction https://doi.org/10.1111/add.15696 
Qu Y, Kim K-H, Szulejko JE. (2018) The effect of flavor content in e-liquids on e-cigarette emissions of carbonyl compounds. Environmental Research, 166: 324-333. https://doi.org/10.1016/j.envres.2018.06.013

Sinclair HK, Bond CM, Stead LF (2004). Community pharmacy personnel interventions for smoking cessation. Cochrane Database of Systematic Reviews. 1L CD0033698.

DOI: https://doi.org/10.1002/14651858.CD003698.pub2

Smoking Toolkit (2021) https://smokinginengland.info/graphs/top-line-findings

Soar K (2021) A retrospective community clinical study to evaluate smoking cessation outcomes by offering smokers a disposable e-cigarette alongside one NRT presentation through a community pharmacy stop smoking service. http://dx.doi.org/10.13140/RG.2.2.13721.13927

Soar K, Kimber C, McRobbie H \& Dawkins LE (2018). Nicotine absorption from e-cigarettes over 12 months. Addictive Behaviours. Available online 21 July 2018 https://doi.org/10.1016/j.addbeh.2018.07.019

Thirlway F (2019) Nicotine addiction as a moral problem Barriers to e-cigarette use for smoking cessation in two working-class areas in Northern England. Social Science \& Medicine, 238:112498 